Western University

Scholarship@Western

Brain and Mind Institute Researchers'

Publications

Brain and Mind Institute

$9-2016$

\title{
Probing the nature of deficits in the 'Approximate Number System' in children with persistent Developmental Dyscalculia
}

\author{
S Bugden \\ Numerical Cognition Laboratory, Department of Psychology, The Brain and Mind Institute, The University \\ of Western Ontario, Canada. \\ Daniel Ansari \\ Numerical Cognition Laboratory, Department of Psychology, The Brain and Mind Institute, The University \\ of Western Ontario, Canada.
}

Follow this and additional works at: https://ir.lib.uwo.ca/brainpub

Part of the Neurosciences Commons, and the Psychology Commons

\section{Citation of this paper:}

Bugden, S and Ansari, Daniel, "Probing the nature of deficits in the 'Approximate Number System' in children with persistent Developmental Dyscalculia" (2016). Brain and Mind Institute Researchers' Publications. 56.

https://ir.lib.uwo.ca/brainpub/56 


\title{
PAPER
}

\section{Probing the nature of deficits in the 'Approximate Number System' in children with persistent Developmental Dyscalculia}

\section{Stephanie Bugden and Daniel Ansari}

\author{
Numerical Cognition Laboratory, Department of Psychology, The Brain and Mind Institute, The University of Western Ontario, Canada
}

\begin{abstract}
In the present study we examined whether children with Developmental Dyscalculia (DD) exhibit a deficit in the so-called 'Approximate Number System' (ANS). To do so, we examined a group of elementary school children who demonstrated persistent low math achievement over 4 years and compared them to typically developing (TD), aged-matched controls. The integrity of the ANS was measured using the Panamath (www.panamath.org) non-symbolic numerical discrimination test. Children with DD demonstrated imprecise ANS acuity indexed by larger Weber fraction (w) compared to TD controls. Given recent findings showing that non-symbolic numerical discrimination is affected by visual parameters, we went further and investigated whether children performed differently on trials on which number of dots and their overall area were either congruent or incongruent with each other. This analysis revealed that differences in $w$ were only found between DD and TD children on the incongruent trials. In addition, visuo-spatial working memory strongly predicts individual differences in ANS acuity (w) during the incongruent trials. Thus the purported ANS deficit in DD can be explained by a difficulty in extracting number from an array of dots when area is anti-correlated with number. These data highlight the role of visuo-spatial working memory during the extraction process, and demonstrate that close attention needs to be paid to perceptual processes invoked by tasks thought to represent measures of the ANS.
\end{abstract}

\section{Research highlights}

- Children with persistent DD demonstrated greater ANS deficits when visual perceptual cues were incongruent with numerical magnitude, but did not show any deficits when visual perceptual cues were congruent with numerical magnitude.

- ANS deficits in children with DD were driven by their inability to inhibit the visual perceptual cues of the dot stimuli to choose the numerically larger quantity.

- Individual differences in visuo-spatial working memory predict performance during incongruent trials in children with persistent DD.

- These findings suggest that non-symbolic numerical discrimination tasks, which were previously thought to assess the integrity of the ANS, are complex and recruit different cognitive processes and strategies across different trial types.

\section{Introduction}

Arithmetic skills are important for children to master early in elementary school for later school and life success (Duncan, Dowsett, Claessens, Magnuson, Huston et al., 2007; Romano, Babchishin, Pagani \& Kohen, 2010); however, for children with Developmental Dyscalculia (DD), learning simple arithmetic is laborious and problematic. DD is a specific learning disorder that is characterized by a persistent impairment in processing numerical information and learning arithmetic facts (Diagnostic and Statistical Manual of Mental Disorders-V; APA, 2013). Children with DD have severe difficulties executing calculation procedures, often relying on immature strategies when they cannot solidify arithmetic facts in long-term memory (Geary, 1993). The underlying cognitive and neural manifestations leading to poor arithmetic performance in children with DD are not well understood (Price \& Ansari, 2013). Furthermore,

Address for correspondence: Daniel Ansari, Numerical Cognition Laboratory, Department of Psychology \& The Brain and Mind Institute, The University of Western Ontario, Westminster Hall, London, ON N6A 3K7, Canada; e-mail: daniel.ansari@uwo.ca 
gaps in our knowledge about the core deficits and characteristics of children with DD have led to inconsistencies in defining and identifying children with DD (Mazzocco \& Myers, 2003).

One dominant proposal is that DD is caused by a core deficit in the so-called 'Approximate Number System' (ANS; Piazza, Facoetti, Trussardi, Berteletti, Conte et al., 2010; Piazza, 2010), a system responsible for manipulating and discriminating approximate numerical quantities (Dehaene, 1997, 2007; Wilson \& Dehaene, 2007). The ANS is thought to be a phylogenetic precursor to developing exact symbolic representations (e.g. number words and Arabic numerals), which enables children to perform basic arithmetic problems and higher order mathematics (Piazza, 2010). Consequently, deficiencies in the ANS would lead to imprecise symbolic representations and poor arithmetic knowledge.

The ANS is commonly assessed using a non-symbolic numerical discrimination task where children are asked to choose the numerically larger dot array as quickly and accurately as they can without counting. Response times and accuracy measures are used as indices for the precision of the ANS. As the numerical distance between the two dot arrays decreases, reaction time and error rates increase - this is referred to as the numerical distance effect (NDE) (Moyer \& Landauer, 1967; Sekuler \& Mierkiewicz, 1977). The numerical ratio effect (NRE) is a complementary effect that takes into account the numerical ratio between the compared dot arrays. The NDE and the NRE have been explained by recourse to models of numerical representation which postulate that magnitudes are represented on a hypothetical internal mental number line where numerical values activate a Gaussian distribution, thus creating overlapping distributions of numbers that are separated by a relatively small numerical distance/have a large numerical ratio (Dehaene \& Cohen, 1995; Dehaene, 1997; Gallistel \& Gelman, 2000). These representations are thought to be analogue and therefore imprecise. The parameters of the Gaussian distribution specify the nature and precision of numerical representations. The Weber fraction (w), which is an index of 'number acuity', is the standard deviation of the estimated Gaussian distribution of the internal representation, and it signifies the degree of precision and the amount of error in one's quantity representations (Halberda, Mazzocco \& Feigenson, 2008). More specifically, as $w$ increases, the noise of the internal representations increases whereby the discrimination of numerical magnitudes close to one another becomes more difficult (Dehaene, 2003, 2007). Therefore, $w$ is a psychophysical model indexing the underlying internal representation of numerical magnitude. Better performance on the non-symbolic numerical discrimination task is marked by a smaller $w$, which is indicative of a more precise internal representation of numerical magnitude (Halberda et al., 2008). There is evidence to suggest that individual differences in $w$ predict variability in symbolic mathematical achievement, supporting the notion that precise numerical magnitude representations are associated with higher mathematical abilities in typically developing individuals (Halberda et al., 2008; Libertus, Feigenson \& Halberda, 2013).

The first evidence supporting the ANS core deficit theory in DD was obtained by Piazza et al. (2010). These authors found that school-aged children with DD demonstrated severely impaired numerical acuity (as indexed by $w$ ) on a non-symbolic numerical discrimination task in comparison to a group of typically developing peers. More specifically, children with DD obtained $w$ scores equivalent to 5-year-old typically developing children, suggesting that their quantity representations are severely delayed. ANS acuity deficits in children with DD were further corroborated by a number of studies (Mazzocco, Feigenson \& Halberda, 2011; Mussolin, Meijas \& Noel, 2010; Price, Holloway, Räsänen, Vesterinen \& Ansari, 2007). In contrast to these findings, some researchers have failed to find performance differences on the non-symbolic numerical discrimination task between children with DD and their typically developing peers (DeSmedt \& Gilmore, 2011; Rousselle \& Noël, 2007).

The finding of lower ANS acuity in individuals with DD has been taken to reflect the impairment of the internal representation of numerical magnitude (i.e. a core representational deficit). However, recent research has suggested that processes other than those attributable to the internal, approximate representation of numerical magnitude influence performance indicators, such as $w$ scores on non-symbolic numerical magnitude discrimination tasks. Specifically, researchers have begun to examine how the visual properties of the dot stimuli impact numerical discriminations. During non-symbolic numerical discrimination, participants can rely on nonnumerical cues such as the size of the individual dots or the total surface area of dots to select the numerically larger dot array. Therefore, to ensure that participants do not use superficial non-numerical cues to choose the numerically larger dot array, researchers commonly use various methods to control for dot size, density and area. The most common method to control for visual parameters is to develop stimuli where the sizes of the dot arrays are either negatively or positively correlated with the larger number in the pair, and to present participants with both trial types to ensure that non-numerical variables are not a reliable cue in non-symbolic numerical 
magnitude discrimination tasks. For example, dot pairs where the more numerous dot array also occupies a larger area are congruent trials, whereas, pairs of dots where the more numerous dot array occupies a smaller area are referred to as incongruent trials. These trials are incongruent because participants are required to ignore the visual size of the dots in order to select the numerically larger dot array. It is important to note that there is no perfect way to control for non-numerical parameters in non-symbolic numerical discrimination (Gebuis \& Reynvoet, 2012). During any given trial, participants can rely on different non-numerical cues to inform their decision; more specifically, on trials where the total surface area are equated, participants can use individual item size to make a response and vice versa.

Furthermore, recent evidence has suggested that the way in which numerical and non-numerical dimensions co-vary affects the strength of the correlation between symbolic math achievement and ANS acuity in typically developing children and adults (Fuhs \& McNeil, 2013; Gilmore, Attridge, Clayton, Cragg, Johnson et al., 2013; Szücs, Nobes, Devine, Gabriel \& Gebuis, 2013b). Specifically, Gilmore and colleagues found that only performance on the incongruent trials (in which the less numerous dot array occupies the larger stimulus area) of the non-symbolic numerical discrimination task was significantly related to symbolic math achievement. In other words, only when children had to resolve a conflict between number and stimulus area did performance account for individual differences in math achievement. Moreover, when non-numerical inhibition scores were controlled for, the relationship became non-significant, suggesting that incongruent trials tap into inhibitory control mechanisms which in turn are correlated with math achievement.

The findings by Gilmore et al. (2013) suggest that the commonly found relationship between math achievement and $w$ is not specific to numerical acuity, but is driven by the relationship between performance on the incongruent trials and individual differences in inhibitory control. This conclusion is also supported by a set of findings presented by Fuhs and McNeil (2013). These authors found that ANS proficiency in preschool children during the incongruent trials (surface area was inversely related to numerical magnitude) predicted math achievement. However, consistent with the findings of Gilmore and colleagues, this association was rendered non-significant once inhibitory control was taken into account. Based on this evidence it has been contended that inhibitory control likely plays a key role in selecting the numerically larger dot array during incongruent trials and, therefore, affects the relationship between ANS acuity and arithmetic achievement in typically developing populations. Furthermore, these findings suggest that performance on tasks used to index the ANS is influenced by the covariation of numerical and non-numerical dimensions, which in turn modulates the relationship between measures of ANS and math achievement.

To date, only a few studies have investigated the effect of non-numerical variables on the non-symbolic numerical magnitude processing in children with DD. In one study, Mussolin et al. (2010) found that children with DD were more sensitive to surface and density cues of the dot stimuli. Specifically, these authors found a trend whereby children with DD made more errors than their typically developing peers when the surface area was incongruent with the number of sticks. A larger congruency effect was observed in a sample of children with DD compared to typical controls in a study conducted by Szucs, Devine, Soltesz, Nobes \& Gabriel (2013a). In addition, Defever, Reynvoet and Gebuis (2011) found that children with and without DD made more errors on non-symbolic numerical magnitude discrimination task trials where the surface area and density of the dots were incongruent with numerical magnitude. Against the background of these findings, the authors suggested that non-symbolic numerical discrimination does not reflect pure numerical processing, but evokes, at least in part, visual processing strategies (Defever et al., 2011). However, from these data, it remains ambiguous what specific underlying mechanisms or strategies are employed by both typically developing and atypically developing populations during non-symbolic discrimination. Furthermore, it is not clear whether children with DD are significantly more affected by the conflicts between numerical and non-numerical variables in non-symbolic numerical magnitude discrimination tasks.

In light of these findings, it remains an open question whether children with DD have an impaired approximate number system which may lead to arithmetic deficits in school, or whether performance differences on nonsymbolic numerical magnitude processing tasks are caused by differences in the way in which DD and TD children process numerical and non-numerical stimulus parameters.

Working memory abilities have been found to predict individual differences in arithmetic achievement in typically developing individuals (Ashkenazi, Rosenberg-Lee, Metcalfe, Swigart \& Menon, 2013; Nath \& Szücs, 2014; Sowinski, LeFevre, Skwarchuk, Kamawar, Bisanz et al., 2015; Swanson, 2004). Moreover, the causal role of working memory in characterizing DD has been a source of controversy within the literature. Some studies have observed working memory deficits in children with DD (Geary \& Brown, 1991; Geary, Hoard, Byrd-Craven \& 
DeSoto, 2004; McLean \& Hitch, 1999), while other studies have found no working memory deficits compared to typically developing controls (Landerl, Bevan \& Butterworth, 2004). To further understand the inconsistent findings, several studies have examined the specific contributions of different working memory constructs and tasks. For example, Passolunnghi and Mammeralla (2012) found that only children with persistent and severe difficulties solving mathematical word problems had impairments in complex visuo-spatial working memory tasks where high attentional control was necessary to complete the tasks. They were able to complete a visual memory recognition task at the same level as typically developing controls. In addition, Szucs et al., (2013a) found that children with DD showed greater impairments in visuo-spatial working memory and visuo-spatial short-term memory, as well as inhibition, compared to typically developing controls. In contrast, children with DD did not reveal a deficit on verbal working memory or verbal short-term memory tasks compared to typically developing controls. Taken together, these data suggest that children with DD have demonstrated specific impairments in visuo-spatial working memory (Ashkenazi et al., 2013; McLean \& Hitch, 1999). It is plausible that an impaired visuospatial working memory system in children with DD would lead to greater difficulties holding and manipulating numerical representations during arithmetic tasks.

It is evident from the above literature review that current findings regarding the ANS in the DD literature are contradictory, and there is no clear conclusion as to what causes DD. Furthermore, there are no universally agreed upon criteria for diagnosing children with DD, and as a result, it is difficult for researchers to make conclusions about what underlying cognitive mechanisms impair their ability to learn basic arithmetic. Some studies have included samples with milder forms of math deficits (Geary et al., 2004; Jordan, Hanich \& Kaplan, 2003), while others use more strict criteria, for example Mazzocco and colleagues limited their sample to children who obtained a math achievement score below the 10th percentile (Mazzocco \& Myers, 2003; Mazzocco, Devlin \& McKenney, 2008). Importantly in the context of the present investigation of ANS deficits in DD, it was only children with DD who met the criteria for severe and persistent math deficits that demonstrated impairments in the ANS, compared to children who had low math achievement (e.g. 11th-35th percentile on math achievement) but did not differ from typically developing children in terms of their ANS acuity. Given that mathematics abilities vary over time (Mazzocco \& Myers, 2003), it has been proposed that research studies impose a stability criterion to ensure that children with
DD are demonstrating persistent arithmetic impairments reducing the number of false positives within a DD sample (Mazzocco \& Räsänen, 2013). This is further supported by the recently published Diagnostic Statistical Manual (American Psychological Association, 2013) requiring symptoms of severe mathematical deficits to be persistent over time to meet the criteria for DD.

The aim of the current study was to investigate differences in the ANS in children with DD characterized by a stable deficit on standardized tests of math achievement compared to typically developing age-matched children. In addition, the effect of the congruency of the visual perceptual and numerical parameters during a non-symbolic numerical discrimination task will be examined between children with and without DD. To elucidate the underlying cognitive mechanisms engaged during non-symbolic discrimination, we explored the relationship between working memory and performance during both the incongruent and congruent trials.

The integrity of the ANS in children with and without DD was evaluated using the Panamath program (Halberda et al., 2008), which is a non-symbolic numerical discrimination task developed to assess the precision of the ANS, and is published online (www.panamath.org) for research and public use. In this program, nonnumerical parameters are controlled such that in half of the trials, the average size of dots of the more numerous dot array contained the smaller sized dots (incongruent trials). On the other half of the trials, the total area of each dot array was proportional to the total number of dots in the larger array (congruent trials). Previous studies have found that children with DD have imprecise approximate numerical representations compared to typically developing children when analyzing the entirety of the non-symbolic numerical discrimination task (both congruent and incongruent trials). Therefore, consistent with previous research (Mazzocco et al., 2011; Piazza et al., 2010), it was hypothesized that children with DD would exhibit imprecise ANS acuity as indicated by a larger $w$ or greater errors compared to typically developing children.

However, as discussed above, recent findings have questioned the precise cognitive mechanisms involved in discriminating between approximate quantities during trials where non-numerical parameters are incongruent with the larger numerosity (Fuhs \& McNeil, 2013; Gilmore et al., 2013; Szucs et al., 2013b). Given these findings, we hypothesize that if children with DD have a pure domain-specific impairment in the ANS, they would demonstrate higher $w$ and greater errors on both incongruent and congruent trials compared to typically developing children. In other words, if the Panamath non-symbolic numerical discrimination is a pure measure 
of approximate numerical abilities, then group differences as a function of the congruency of the visual perceptual cues and numerical dimension would not be expected. However, if deficits on the non-symbolic numerical discrimination task are driven by difficulties in processing the conflict between numerical and nonnumerical stimulus attributes, we would expect to find a larger size congruity effect in children with DD compared to typically developing children.

To scrutinize possible mechanistic processes subserving approximate numerical discriminations, we examined whether verbal or visuo-spatial working memory predict individual differences in ANS acuity. Given the ambiguities surrounding the involvement of working memory during arithmetic processing in children with DD, correlation analyses were conducted independently for children with DD and typically developing controls. These analyses were conducted separately between groups to elucidate whether children with DD recruit different cognitive processes during the discrimination of incongruent and congruent dot stimuli compared to typically developing children. Following the results presented by Nath and Szücs (2014), as well as Szucs et al. (2013a, 2014), we hypothesize that visuo-spatial working memory and measures of ANS acuity are significantly related, whereas, no such correlations are expected with verbal working memory. Furthermore, it is thought that visuo-spatial working memory (to a greater extent than verbal working memory) may be required to disentangle the visual perceptual cues from the quantitative information to select the correct response.

\section{Method}

\section{Participants}

Participants from the current study were recruited from a longitudinal screening study conducted in schools across the local school board and surrounding area (see Archibald, Cardy, Joanisse \& Ansari, 2013, for a complete description of the original sample).

\section{Participant selection criteria}

Developmental dyscalculia. Fifteen children with Developmental Dyscalculia (Mean age $=12.36, S D=$ 1.20; range: $9.44-13.68$ years) were included in the present study (11 boys, 4 girls). To meet our criteria for DD, children had to demonstrate stable low math impairments on Math Fluency and Math Calculation subtests (timed and untimed tests of basic arithmetic) from the Woodcock Johnson-III standardized tests of math achievement (Woodcock, McGrew \& Mather, 2001) over four years. First, children were recruited for the current study if they obtained one standard deviation below the mean on both the Math Fluency and Math Calculation subtests during screening as well as subsequent testing sessions (spring 2010 and spring 2011; see Figure 1). There was one child with DD who obtained a standard score of 90 and 88 on the Math Fluency subtest during screening and the spring 2012 session, and there was one child with DD who obtained a standard score of 97 on the Math Fluency subtest during screening. However, both children obtained standard scores on both the Math Fluency and Math Calculation subtests below 85 during the remaining testing sessions. Children who were selected based on these criteria were recruited for follow-up testing during the spring of 2012 and fall of 2013, at which time the non-symbolic numerical discrimination task was administered (see Figure 1). At the time of the final testing session in fall 2013, children with DD persistently performed below average on the standardized tests of math achievement. However, children with DD demonstrated variable performance on the Reading Fluency subtest, as well as verbal and visuospatial working memory measures when collected during the spring testing sessions. There were five children who had a stable low reading deficit on the Reading Fluency subtest across all testing sessions, and therefore, may

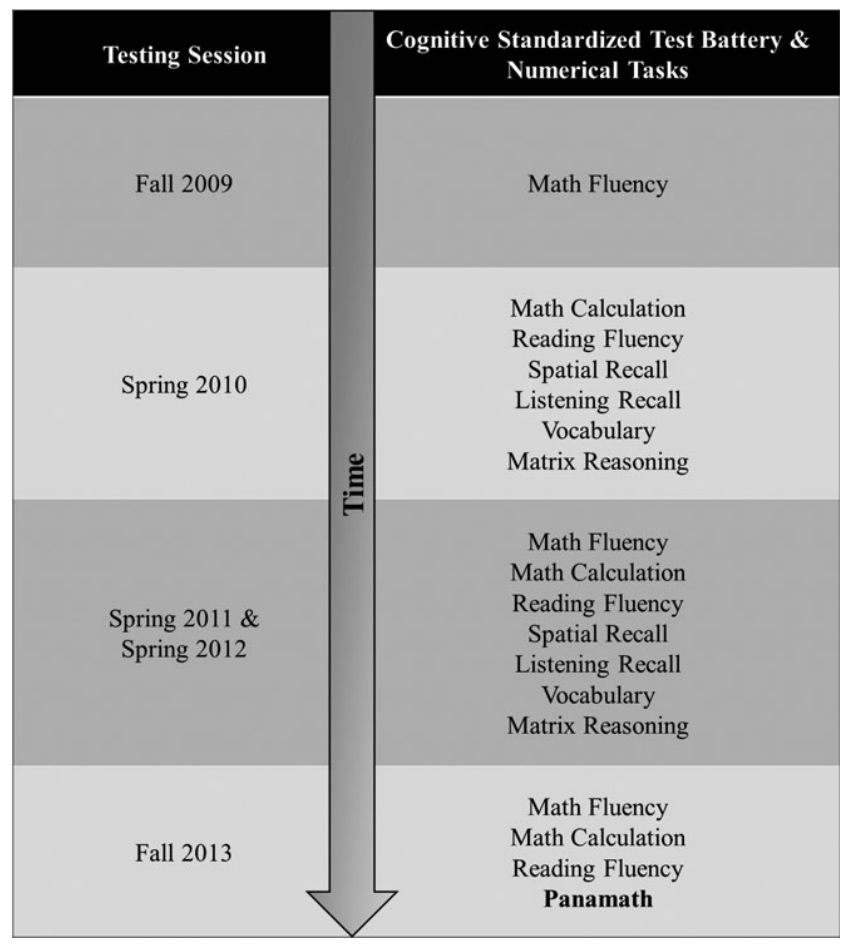

Figure 1 Testing sessions. 
have comorbid dyslexia (a specific reading learning disability). However, evidence has demonstrated that processing numerical magnitudes is not further impaired by a reading learning disability (Hanich, Jordan, Kaplan \& Dick, 2001; Landerl et al., 2004). In accordance with the DSM-V criteria for DD, all children demonstrated a stable standardized IQ score greater than 70 , and parents reported their children as having various academic difficulties in school (APA, 2013) (see Table 1 for a summary of descriptives).

Typically developing control group. The control group consisted of 15 typically developing children who were age matched to the DD group (Mean age $=11.78, S D=$ .82 ; range $10.32-13.37$ years; 8 boys, 7 girls). There were no significant differences of age found between DD and typical groups, $t(28)=-1.54, p=.14$ (see Table 1). Children were recruited to be in the typically developing group if they demonstrated persistent typical performance or above ( $>85$ standard score) on both subtests of arithmetic achievement, as well as working memory and reading fluency and IQ during the screening session and the spring sessions in 2010 and 2011. Similarly to the DD children, typically developing children were recruited for the sessions in spring of 2012 and fall of 2013 if they obtained a standard score of above 85 on measures of arithmetic achievement, reading and working memory. The typically developing group demonstrated stable IQ, working memory, arithmetic, and reading achievement scores within the normal range and above over all testing sessions. However, during the fall of 2013, there were three typically developing children who performed just below the cut-off criteria of 85 on both Math Fluency and Math Calculation subtests.

\section{Cognitive performance across groups}

Composite scores of Math Fluency, Calculation, Reading Fluency, verbal working memory, visuo-spatial WM and full scale IQ were calculated by computing the mean standard score for measures collected across all testing sessions. Subsequent analyses including standardized scores of cognitive performance (e.g. Math achievement) were conducted using the mean composite scores representing their average ability on the specific measure of interest. For example, the math average composite score was calculated using standard scores collected from the Math Fluency and Math Calculation subtests for all testing sessions. Given that children with DD demonstrated variable performance on measures of reading and working memory, a mixed ANOVA with measure (math, reading, IQ, verbal $\mathrm{WM}$ and visuo-spatial $\mathrm{WM}$ ) and Group (DD, TD) was performed to investigate differ- ences in performance on standardized tests. Mauchly's test of sphericity was not violated, and therefore no corrections for inflated $p$-values were applied. An interaction was found between measure and group $[F(4,112)$ $=5.03, p=.001, \eta^{2}=.39$, with an observed power of .84$]$. In addition, the group difference on mathematical measures produced the largest effect sizes, further highlighting the severe mathematical deficits in the present sample of children with DD (see Table 1 for the effect sizes). These findings demonstrate that although children with DD have poor performance on reading, IQ and working memory, they have the greatest impairment in mathematical performance (see Figure 2). ${ }^{1}$

\section{Materials}

Standardized tests of cognitive performance

Mathematical skills. The Math Calculation and Math Fluency subtests from the Woodcock-Johnson standardized tests of achievement (Woodcock et al., 2001) were administered to each participant. First, the Math Calculation subtest was administered to assess basic arithmetic skills. This test begins with simple addition and subtraction problems and becomes progressively more difficult. Participants had no time constraints and were asked to inform the experimenter when they had finished. Second, the Math Fluency subtest assessed participants' ability to solve as many simple arithmetic problems as possible in 3 minutes without making any errors.

\footnotetext{
${ }^{1}$ Multiple two-way ANOVAs that included math composite scores were conducted to establish the locus of the interaction between measures, including all cognitive tests, and group. With math composite scores and visuo-spatial working memory included in the model, a significant group by measure interaction was found indicating that math performance was significantly lower than visuo-spatial working memory in the sample of children with DD, $F(1,28)=16.83, p<.001$, $\eta^{2}=.38$. In addition, a significant interaction between group and measure, including math composite scores and verbal working memory, was found, $F(1,28)=5.44, p=.027, \eta^{2}=.16$, as well as a marginal significant interaction between measure and group when math and IQ were included in the model, $F(1,28)=3.06, p=.09, \eta^{2}=.10$. These findings demonstrate that math performance was significantly more impaired in children with DD compared to their working memory ability and IQ. However, there was no significant interaction found between group and measures when math and reading achievement were included demonstrating that children with DD also exhibited severe reading performance when compared to other cognitive measures $(F<$ 1). Taken together, these findings demonstrate that although children with DD have poor performance on reading, IQ and working memory, they have the greatest impairment in mathematical performance.
} 
Table 1 Mean cognitive performance on standardized measures across all testing sessions in both persistent DD and typically developing samples

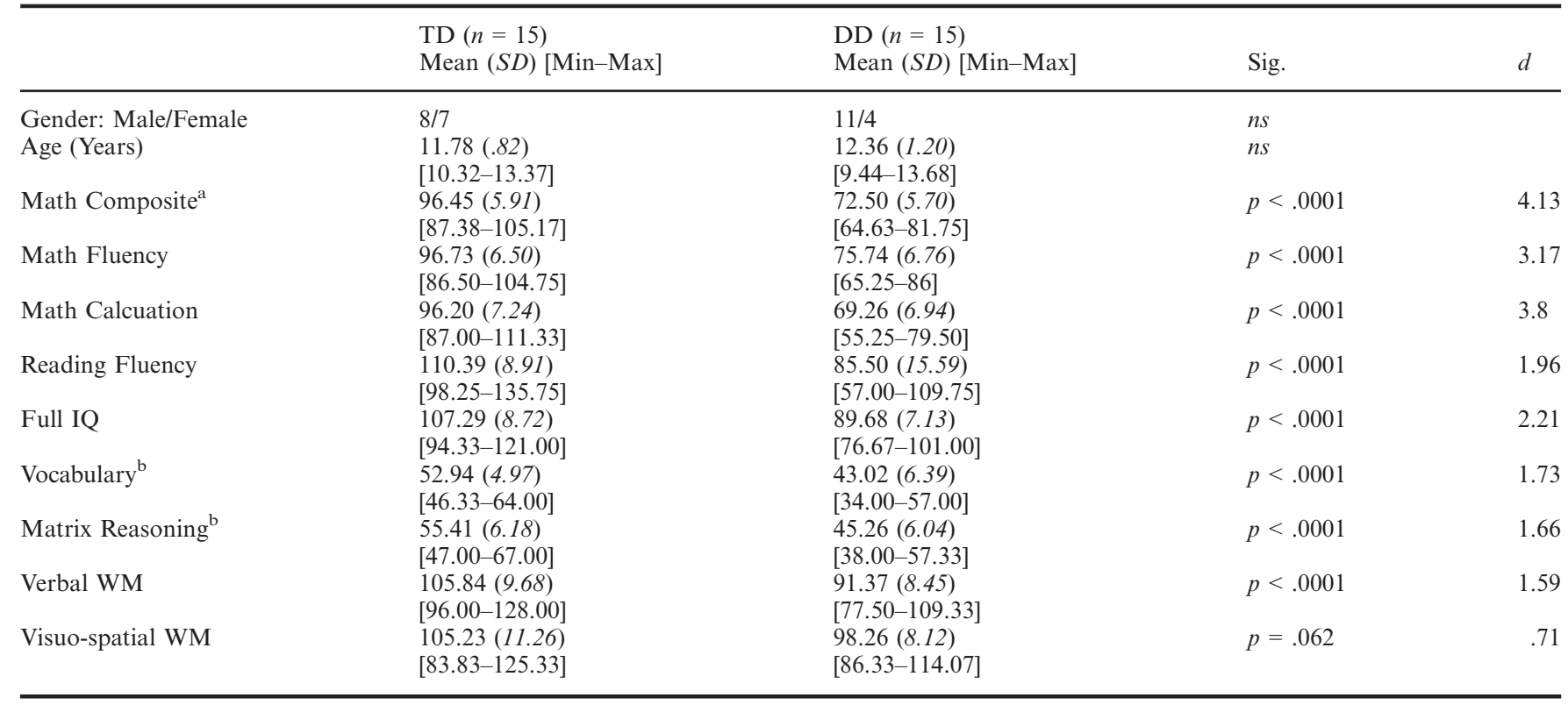

Note: $\mathrm{TD}=$ typically developing; $\mathrm{DD}=$ developmental dyscalculia; $\mathrm{WM}=$ working memory. ${ }^{\mathrm{a}}$ Math Composite scores for each participant were

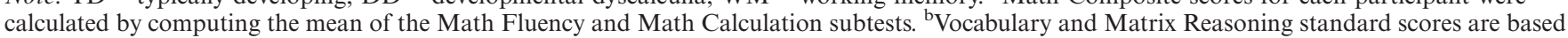
on a normal distribution with a mean of 50 and a standard deviation of 10 .

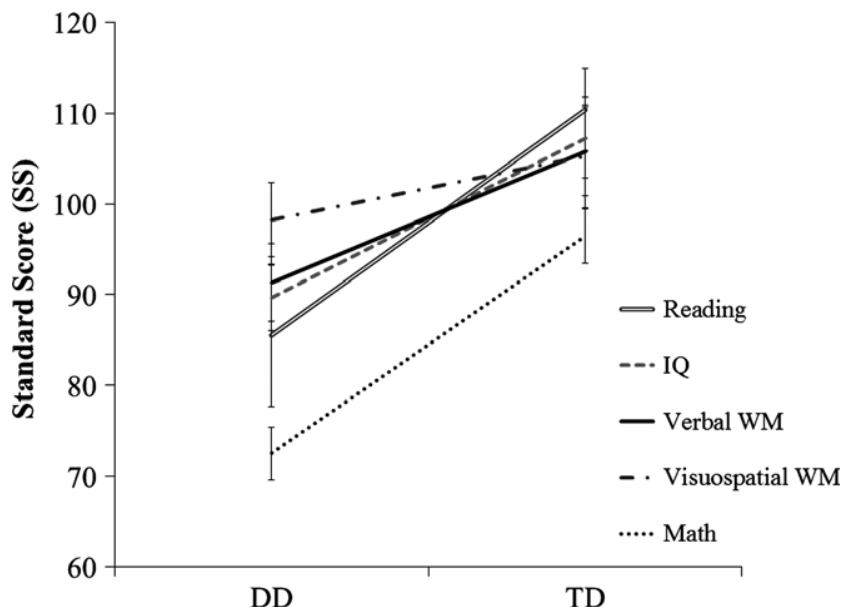

Figure 2 Cognitive Measure $\times$ Group interaction demonstrating that children with $D D$ have the greatest impairment in math performance. Error bars represent 95\% confidence intervals.

Reading skills. The Reading Fluency subtest from the Woodcock-Johnson-III (Woodcock et al., 2001) was administered to measure participants' ability to quickly read simple sentences and answer yes/no questions about each sentence. Participants were asked to read as many sentences as possible in 3 minutes.
Working memory skills. Two subtests from the Automated Working Memory Assessment (AWMA; Alloway, 2007) were administered to assess visuospatial and verbal working memory abilities. The Spatial Recall subtest required participants to mentally rotate shapes while maintaining and remembering the location of a red dot. The Listening recall subtest required participants to process the veracity of a simple sentence while remembering the final word. Both subtests increased in difficulty as participants had to hold more items in memory.

Intelligence. Children completed two subtests from the Wechsler Abbreviated Scale of Intelligence (Wechsler, 1999). The Vocabulary subtest was administered to assess verbal intelligence. Children were asked to provide definitions for words that became increasingly more difficult. Children reached ceiling when they could not produce the correct definitions for three consecutive words. The Matrix Reasoning subtest was administered to measure non-verbal intelligence where children had to select an image to complete the presented pattern.

Non-symbolic discrimination: Panamath

ANS acuity was assessed using the Panamath version 1.22 software (Halberda et al., 2008; http://pana- 
math.org) available online. Panamath is a non-symbolic discrimination task where yellow dot arrays and blue dot arrays are presented simultaneously side by side on a computer screen. Participants were asked to select the numerically larger dot array as quickly and as accurately as possible by pressing the respective button on a laptop. Stimuli display times were tailored to the age of the participant. Stimuli were presented on the computer screen for $1506 \mathrm{~ms}, 1382 \mathrm{~ms}, 1269 \mathrm{~ms}, 1165 \mathrm{~ms}$, or $1071 \mathrm{~ms}$ for participants, who were $9,10,11,12$ or 13 years of age, respectively. Each trial was followed by a backward mask of yellow and blue white noise (e.g. pixelations) and then a grey screen. Participants could respond during or after the presentation of the dot arrays. Following a response, a fixation cross appeared until the participant pressed the space bar to display the next trial. The number of dots presented in the dot arrays ranged from five to 21 . The level of difficulty was manipulated by varying the ratio between the left and right dot arrays; the ratios were 3:8, 2:3, 4:5, and 7:8. Half of the trials were congruent (non size-controlled), meaning that both sets of colored dots were proportional to the number of dots within the array. During these trials, the area or the amount of color in the larger dot array was congruent with its numerosity. The other half of the trials was incongruent (size-controlled), meaning that the proportion of area occupied by each colored dot array was equal, and therefore, the size of the individual dots was negatively correlated with numerosity. For these trials, children could not select the larger dot array by relying on the amount of color occupying space on the computer screen (see Figure 3). Panamath was administered for a total of 5 minutes, and depending on each individual's speed of response, the number of trials varied by participant. The total number of trials completed by participants ranged from 104 to 128 trials, with younger participants completing fewer trials.

A Weber fraction was generated by the Panamath software for each participant using the model employed in Halberda et al. (2008). In addition, a separate $w$ was calculated for each participant for congruent and incon-

Size Control: Incongruent
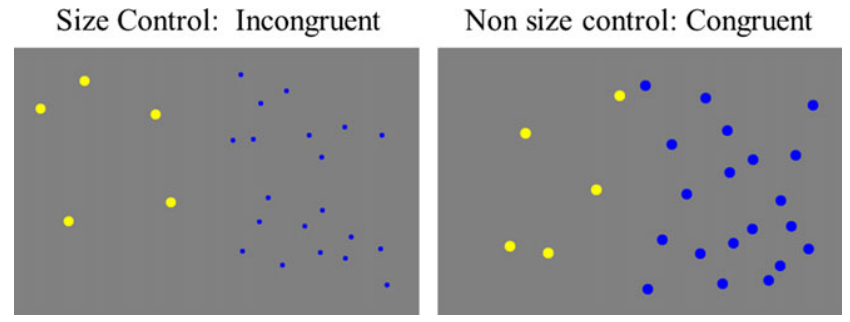

Figure 3 An example of incongruent and congruent stimuli administered in the Panamath task. gruent trials separately (see Panamath.org; Halberda et al., 2008, for a complete description of the modeling parameters for obtaining individual Weber fractions).

\section{Procedure}

During four previous visits, each child completed the standardized battery of cognitive tests measuring math, reading, working memory skills and intelligence (see Figure 1). During the fifth visit, children were tested individually in a quiet laboratory testing room in the university, where participants completed the Reading Fluency, Math Fluency and Calculation subtests from the Woodcock Johnson tests of achievement (Woodcock et al., 2001). Following the standardized tests, participants completed the Panamath non-symbolic discrimination task (and other tasks not reported here) (see Figure 1 for a time line of testing sessions and standardized measures). This session lasted approximately one hour.

\section{Results}

\section{Weber fraction $(w)$}

To investigate whether the congruity of the dot stimuli influenced performance differently in our sample of persistent DD and typically developing children, a 2 (incongruent, congruent) $\times 2$ (DD, Typical) mixed factorial ANOVA was conducted on $w$. We found a significant main effect of group, $F(1,28)=6.24, p=.019$, $\eta^{2}=.18$, a significant main effect of congruity, $F(1,28)=$ $11.82, p=.002, \eta^{2}=.23$, as well as a significant interaction between group and congruity, $F(1,28)=$ $5.68, p=.02, \eta^{2}=.17$. To further explore the locus of the interaction, Bonferroni-corrected independent samples $t$ tests were conducted on $w$ for the incongruent and congruent trials between groups (significance was reached if $p<.0125$ ). Means, 95\% confidence interval bounds for each group mean, as well as the standard deviations, are reported for each $t$-test in parentheses. The results from these analyses indicate that there was a significant difference between children with DD and typically developing children during the incongruent trials, $t(15.72)=-.2 .80, p=.013, d=1.41$; equal variances not assumed, $F(1,28)=10.14, p=.004$; however, there was no significant difference between groups during the congruent trials, $t(28)=-1.64, p=.113$, $d=.62$, equal variances assumed, $F(1,28)=2.10, p=$ .16. These findings suggest that differences in ANS acuity were driven by performance during the incongruent trials; specifically, a larger $w$ was found for children 

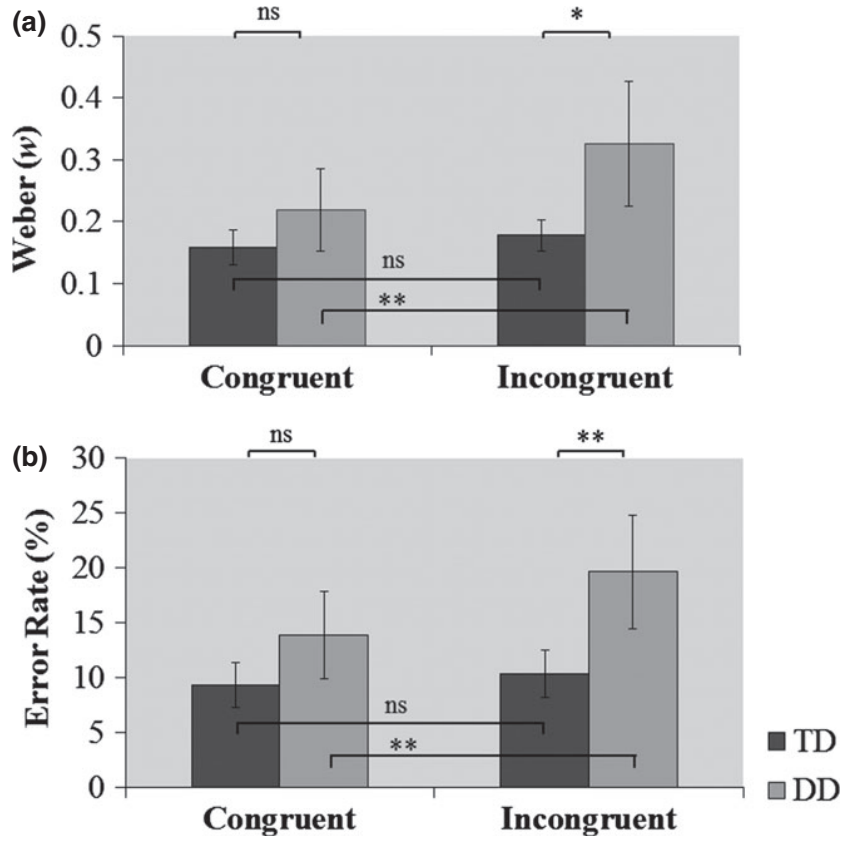

Figure 4 A significant interaction between group and congruency during the Panamath non-symbolic discrimination indicating children with DD were less accurate and precise at choosing the numerically larger dot array in the incongruent trials. (a) Bars represent a larger mean $w$ fraction in children with DD during the incongruent trials compared to typically developing children. (b) Bars represent mean error rates, with greater errors being made by children with DD during the incongruent trials in comparison to typically developing children. Error bars represent 95\% confidence intervals.

with DD $(M=.33 \pm .10, S D=.20)$ compared to typically developing children $(M=.18 \pm .03, S D=.05)$. However, there were no group differences in ANS acuity during the congruent trials (DD: $M=.22 \pm .07, S D=$ .13 ; TD: $M=.16 \pm .03, S D=.06)$. Paired samples $t$-tests were conducted to investigate the simple main effects of congruency within group. There were no significant differences in $w$ between incongruent and congruent trials in typically developing children, $t(14)=$ $-1.30, p=.22, d=.69$. However, children with DD had a significantly larger $w$ during the incongruent trials compared to congruent trials, $t(14)=-3.19, p=.007$, $d=1.71$ (see Figure 4a). The default settings of the Panamath task included presenting the dot stimuli for different display times according to age. Given that previous research has shown that varying display times can influence the estimate of $w$ (Inglis \& Gilmore, 2013), we included age as a covariate in a mixed factorial ANCOVA to examine whether the display times change the outcome of the results (since age and display time are related). We found that the significant interaction between congruency and group remains significant, $F(1$, 27) $=4.13, p=.05, \eta^{2}=.13$, in addition to the main effect of group, $F(1,27)=4.65, p=.04, \eta^{2}=.16$. No other effects were significant when age was included as a covariate.

It has been proposed that comorbidity of dyslexia (RD) and DD results from domain-general deficits in processing speed and working memory that cause greater deficits in mathematical and reading performance in comparison to children with RD or DD alone (Willcutt, Petrill, Wu, Boada, DeFries et al., 2013). We examined whether ANS acuity deficits observed during the incongruent trials were attributable to the severity of having comorbid learning disorders. Five children who demonstrated persistent impairments in reading fluency scores were removed from the analysis in order to examine whether the effect of having comorbid DD and RD (double deficit) were driving poor performance during the incongruent trials. The interaction between congruency of dot stimuli and group remained significant, $F(1$, $23)=6.23, p<.02, \eta^{2}=.21$. The interaction was driven by significant group differences during the incongruent trials, $t(10.52)=-2.48, p<.05, d=1.03$, equal variances not assumed, $F(1,23)=6.35, p=.02$ [Incongruent Trials: DD $(n=10): M=.29 \pm .07, S D=.14$; TD: $M=.17 \pm .03, S D=.05]$ but no significant differences during the congruent trials, $t(23)=-1.14, p=.26$, $d=.48$ [Congruent Trials: DD $(n=10): M=.19 \pm .03$, $S D=.07$; TD: $M=.16 \pm .03, S D=.06]$. In addition, the effects were not altered when the three typically developing children who demonstrated low math scores during the last testing session were removed from the analysis.

\section{Error rates}

Although we reported $w$ in the main analysis above, the same pattern of findings was found using error rates (see Figure 3). When error rates were submitted to a 2 (incongruent, congruent) $\times 2(\mathrm{DD}$, Typical) mixed factorial ANOVA, similar to the $w$ analysis, a significant main effect of group, $F(1,28)=8.27, p=.008, \eta^{2}=.23$, a significant main effect of Congruity, $F(1,28)=11.74$, $p=.002, \eta^{2}=.30$, and a significant interaction between congruity and group, $F(1,28)=5.83, p=.02, \eta^{2}=.17$, were found. To further explore the locus of the interaction, a Bonferroni-corrected independent samples $t$-test was conducted on error rates in the incongruent and congruent trials between groups (significance was reached if $p<.0125)$. The results from these analyses indicated that children with DD $(M=19.64 \pm 5.16, S D=10.19)$ made more errors during the incongruent trials compared to typically developing children $(M=10.38+/-2.13$, 
$S D=4.21), t(18.65)=-3.25, p=.004, d=1.51$; equal variances not assumed, $F(1,28)=6.82, p=.01$; however, there were only marginally significant differences in the congruent trials, $t(28)=-1.96, p=.06, d=.74$, equal variances assumed, $F(1,28)=1.22, p=.30(\mathrm{DD}$ : $M=13.87 \pm 4.00, S D=7.91 ; \mathrm{TD}: M=9.38 \pm 2.06$, $S D=4.07$ ). Paired samples $t$-tests were conducted to investigate the simple main effects of congruency within group. There were no significant differences in error rates between incongruent and congruent trials in typically developing children, $t(14)=-.84, p=.42, d=.45$. However, children with DD had significantly greater error rates during the incongruent trials compared to congruent trials, $t(14)=-3.67, p=.003, d=1.96$. When age was included as a covariate in the mixed factorial ANCOVA, the interaction between congruency and group on error rates remained significant, $F(1,27)=4.65$, $p=.04, \eta^{2}=.15$, as well as the main effect of group, $F(1$, $27)=6.73, p=.02, \eta^{2}=.20$. In agreement with the analysis conducted on $w$, these findings demonstrate that children with DD had greater error rates during incongruent trials, where the individual dot size was incongruent with the numerical magnitude of the larger dot array, compared to performance during the congruent trials, where the dot size was congruent with the numerical magnitude of the larger dot array (see Figure $4 b)$.

\section{Correlational analysis: math achievement}

The relationship between math achievement and performance during the incongruent and congruent trials was examined between both groups. Within the typically developing sample, math achievement (composite of Math Fluency and Calculation) did not significantly correlate with $w$ during the congruent, $r(13)=.07$, $p=.81$ or incongruent trials, $r(13)=.13, p=.66$, or error rates during the congruent trials, $r(13)=.01$, $p=.98$, or incongruent trials, $\mathrm{r}(13)=.14, p=.61$. In contrast, in children with DD, math achievement (composite of Math Fluency and Calculation) significantly correlated with $w$ during the incongruent trials, $r$ $(13)=-.61, p=.02$, but did not correlate with $w$ during the congruent trials, $r(13)=-.49, p=.07$. Math achievement correlated with error rates during both the incongruent trials, $r(13)-.57, p=.03$, and congruent trials, $r(13)-.51, p=.05$. These findings demonstrate that individual differences in math ability correlate with $w$ and error rates for incongruent and congruent trials in children with DD, but not for the typically developing children. This is consistent with the ANOVA analysis demonstrating that the severity of DD is most strongly correlated to the incongruent trials.

\section{Correlational analysis: working memory}

To examine the role of working memory during the discrimination of congruent and incongruent trials of the Panamath task in children with DD as well as typically developing children, a correlation analysis was conducted between the $w$ on the incongruent and congruent trials and verbal and visuo-spatial working memory measures. This analysis was conducted to evaluate whether visuo-spatial working memory abilities modulate performance during non-symbolic discrimination differently in children with DD and typically developing children. Correlations were performed within groups to ensure that correlations were not driven by group differences.

\section{Developmental dyscalculia}

For children with DD, visuo-spatial working memory significantly correlated with $w$ during incongruent trials, as well as congruent trials, $r(13)=-.57, p=.026 ; r(13)=-.52$, $p=.046$, respectively (see Figures 4-5). Error rates during the incongruent trials were significantly correlated with visuo-spatial working memory, $r(13)=-.58, p=.024$; and a marginally significant relationship was found between error rates during congruent trials and visuo-spatial working memory, $r(13)=.49, p=.06$. There were no significant correlations between error rates and $w$ during the incongruent and congruent trials and verbal working memory abilities (see Table 2 for correlation matrix).

\section{Typically developing children}

Visuo-spatial working memory did not significantly correlate with $w$ during the incongruent, $r(13)=-.06$, $p=.82$, and congruent trials, $r(13)=-.03, p=.91$; however, $w$ during the congruent trials significantly correlated with verbal working memory, $r(13)=-.57$, $p=.03$. Error rates during the incongruent trials did not significantly correlate with visuo-spatial working memory, $r(13)=-.08, p=.77$ (see Figure 5). Furthermore, there were no significant correlations between error rates during the congruent trials and visuo-spatial working memory or verbal working memory, $r(13)=-.14$, $p=.63 ; r(13)=-.40, p=.14$, respectively (see Figure 5).

\section{Differences in correlation coefficients}

To examine whether there was a significant difference in the correlation between incongruent $w$ and visuo-spatial working memory in typically developing children $(r=$ $-.06)$ and children with DD $(r=-.57)$, a Fisher's $z$-test was conducted. A one-tailed $z$-test demonstrated that 
Table 2 Correlation matrix

\begin{tabular}{|c|c|c|c|c|c|c|c|}
\hline DD & 1 & 2 & 3 & 4 & 5 & 6 & 7 \\
\hline $\begin{array}{l}\text { 1. Incongruent } w \\
\text { 2. Congruent } w \\
\text { 3. Incongruent ERR } \\
\text { 4. Congruent ERR } \\
\text { 5. Verbal WM } \\
\text { 6. Visuo-spatial WM } \\
\text { 7. Math achievement }{ }^{\mathrm{a}}\end{array}$ & & $\begin{array}{l}.76^{* *} \\
.41 / .92\end{array}$ & $\begin{array}{l}.96 * * \\
.88 / .99 \\
.70^{* *} \\
.29 / .89\end{array}$ & $\begin{array}{l}.85^{* *} \\
.60 / .95 \\
.97^{* *} \\
.91 / .99 \\
.80^{* *} \\
.49 / .93\end{array}$ & $\begin{array}{c}. \mathbf{1 6} \\
-.38 / .62 \\
\mathbf{. 1 7} \\
-.38 / .63 \\
. \mathbf{2 1} \\
-.39 / .65 \\
. \mathbf{1 8} \\
-.37 / .63\end{array}$ & $\begin{array}{l}-.57^{*} \\
-.08 /-.84 \\
-.522^{*} \\
-.01 /-.82 \\
-. .58 * \\
-.10 /-.84 \\
-\mathbf{4 9}^{\dagger} \\
-.80 / .03 \\
-.22 \\
-.66 / .33\end{array}$ & $\begin{array}{l}-.61^{*} \\
-.86 /-.14 \\
-. \mathbf{4 9} \\
-.80 / .03 \\
-.57^{*} \\
-.84 /-.08 \\
-.51^{*} \\
-.81 / .003 \\
. \mathbf{0 5} \\
-.48 / .54 \\
. \mathbf{6 8 *} \\
.26 / .89\end{array}$ \\
\hline TD & 1 & 2 & 3 & 4 & 5 & 6 & 7 \\
\hline
\end{tabular}

Note: $\mathrm{DD}=$ developmental dyscalculia; TD = typically developing children; $w=$ weber fraction; ERR = error rates; WM = working memory. Upper and lower bounds of a 95\% confidence interval are included below the Pearson correlation coefficients, which are in bold. ${ }^{a} \mathrm{Math}$ achievement was computed by calculating the mean standard score for both Math Fluency and Math Calculation subtests. $*=p \leq .05 ; * *=p<.001 ;{ }^{\dagger}=p<.10$.

there was a marginally significant difference between the strength of the relationship between performance during the incongruent trials and visuo-spatial working memory in typically developing children and children with DD $(z=-1.44, p=.07$; two-tailed test $p=.15)$. This difference remained marginally significant when correlation coefficients for the relationship between incongruent error rates and visuo-spatial working memory were submitted to a Fisher's $z$-test $(z=-.1 .43, p=.08$; two-tailed $p=.15)$.

\section{Discussion}

Previous studies have revealed that children with DD perform poorly on non-symbolic number discrimination tasks, such as the Panamath task (Mazzocco et al., 2011; Piazza et al., 2010). These group differences have been postulated to reflect a core representational impairment of numerical magnitude processing, or an impaired Approximate Number System (ANS) in DD. Recent research has found that measures of $w$ and error rates collected from this task are influenced by the relationship between numerical and non-numerical parameters of non-symbolic stimuli in typically developing children (Fuhs \& MacNeil, 2013; Gilmore et al., 2013; Szücs et al., 2013b). These recent data call into question whether group differences on ANS tasks between children with DD and typically developing children can be solely attributable to an impairment of the representations that are thought to drive performance in an ANS task. Alternatively, it may be the processes related to dealing with conflicting numerical and non-numerical cues in non-symbolic numerical magnitude processing tasks that drive group differences between children with and without DD. Accordingly, the present study aimed to elucidate the role of the ANS in characterizing the cognitive deficits in a sample of children with persistent arithmetic impairments. Specifically, we examined whether controlling for visual perceptual parameters, such as area, of the dot stimuli alters measures of ANS acuity and differences therein between children with and without persistent DD. To extend the current body of literature, we examined the relationship between verbal and visuo-spatial working memory and performance 

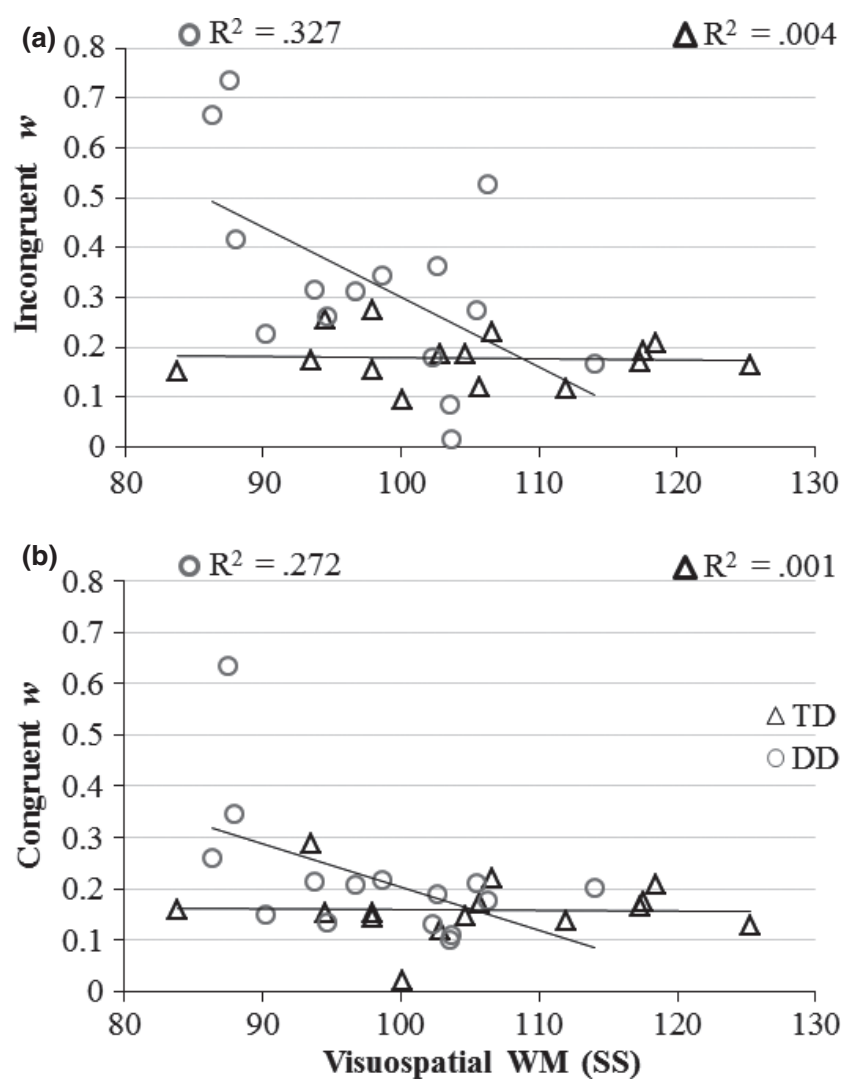

Figure 5 Correlational analyses. (a) The relationship between $w$ during the incongruent trials of the Panamath task and visuospatial WM separately in DD and TD children. (b) The relationship between $w$ during the congruent trials of the Panamath task and visuo-spatial WM in DD and TD children. Note: $w=$ Weber fraction; $W M=$ working memory; $S S=$ standard score; $T D=$ typically developing; $D D=$ developmental dyscalculia.

during the congruent and incongruent trials of the Panamath task within each group. Consistent with Mazzocco and Räsänen (2013), we included a stability criterion in our selection criteria for DD to ensure that children with DD had severe and persistent arithmetic impairments. This methodological strength reduces the probability of including false positive cases as we are confident that the present findings are specific to children with severe arithmetic deficits.

Based on previous findings, we hypothesized that children with persistent DD would demonstrate greater error rates and larger $w$ on the Panamath task compared to typically developing peers (Mazzocco et al., 2011; Piazza et al., 2010). However, given recent findings (Gilmore et al., 2013; McNeil \& Fuhs, 2013; Szücs et al., 2013b), we further predicted that if the approximate number system were truly impaired in children with DD, then they would have greater error rates and $w$ regardless of the congruency of the dot stimuli compared to typically developing peers. In contrast, if domain-general ancillary systems or low-level visual perceptual processes are compromised in children with DD, making it difficult for them to tease apart conflicting numerical and nonnumerical parameters, then performance differences would only be expected when there are conflicts between numerical and non-numerical cues. Specifically, it could be expected that children with DD would demonstrate greater error rates and imprecise $w$ during trials where the total area of dot stimuli is incongruent with the larger numerosity compared to congruent trials.

Consistent with previous research (Mazzocco et al., 2011; Piazza et al., 2010), we found that children with persistent DD demonstrated significantly greater error rates and $w$ compared to typically developing children. However, in contrast to these studies, in which researchers did not examine the effect of congruency on performance, we found that differences between DD and typically developing children were driven by performance during the incongruent trials. These results are consistent with Szucs et al. (2013a); specifically, children with DD demonstrated greater error rates and $w$ during the incongruent trials, where the size of the dot stimuli was anti-correlated with numerical magnitude, compared to their typical age matched peers. In contrast, their ability to discriminate numerical dot arrays remained intact during the congruent trials, where the total area of dot stimuli was positively correlated with numerical magnitude, in relation to their typically developing peers' performance.

These findings reveal that indices commonly used to measure the internal representation of numerical magnitude (e.g. $w$ and error rates) are greatly affected by the methods used to control for visual parameters of dot stimuli and that this affects children with DD to a greater extent than their typically developing peers. These methods are employed to ensure that across all trials of the numerical discrimination task, participants cannot rely solely on visual cues to inform their decision, but forces participants to use numerical cues to discriminate between approximate magnitudes. However, after examining $w$ and error rates separately during different trial types (incongruent and congruent trials) in children with DD, these indices of internal quantity representations clearly change as a function of the congruency of dot stimuli. In other words, having both congruent and incongruent trials does not eliminate the influence of non-numerical variables on numerical magnitude discrimination.

This is consistent with studies investigating the effect of visual perceptual cues in typically developing adults demonstrating that they do not extract number from nonsymbolic stimuli independently of the visual perceptual cues present (Gebuis \& Reynvoet, 2012; Leibovich \& 
Henik, 2014). In the natural environment, it is often the case that individuals rely on visual cues to inform their numerical judgments (Gebuis \& Reynvoet, 2012); but in a laboratory setting, these cues are controlled to isolate numerically specific processes. However, evidence suggests that this is not the case. Instead, individuals engage other cognitive processes and strategies across different trial types to select the numerically larger dot array (Gebuis \& Reynvoet, 2012; Leibovich \& Henik, 2014).

In addition to examining the role of congruency between numerical and non-numerical variables in nonsymbolic number discrimination, we examined the role of working memory as a potential mechanistic candidate for how children with DD discriminate between non-symbolic numerical magnitudes differently from typically developing controls. In particular, we correlated measures of working memory with $w$ collected from the incongruent and congruent trials separately. Correlation analyses were conducted independently for both groups to examine whether children with DD recruited different cognitive processes during the different trial types. Although we did not administer an inhibitory control measure, we hypothesized that visuo-spatial working memory specifically would be required to disambiguate between the conflicting cues during non-symbolic numerical discrimination. Indeed, we found that visuo-spatial working memory negatively correlated with $w$ during the incongruent trials, and marginally correlated with error rates during the congruent trials in children with DD, but not typically developing children. More specifically, children with DD who had lower visuo-spatial working memory abilities had greater difficulty discriminating between non-symbolic numerical magnitudes during the incongruent trials. These relationships were specific to visuo-spatial working memory as there were no significant correlations found between performance on Panamath and verbal working memory. These findings shed light on the qualitative differences between typically developing children and children with DD in the way in which visuo-spatial working memory abilities modulate performance during non-symbolic numerical discrimination, more so during the incongruent trials of the task.

Based on these findings, we can offer three possible interpretations to explain why children with DD demonstrate greater error rates and imprecise $w$ during the incongruent trials and how performance changes as a function of working memory ability in DD but not TD.

First, in light of these data, it is possible that children with DD do not necessarily suffer from an impaired ANS, but experience difficulties with inhibiting the irrelevant non-numerical dimensions during nonsymbolic numerical discrimination. In other words, the deficit does not lie at the representational level, but children with DD exhibit difficulties in accessing the numerical representation when non-numerical cues are interfering with this process. To successfully choose the numerically larger dot array during the incongruent trials (when the size of the dots is negatively correlated with numerosity), participants are required to suppress or inhibit the conflicting visual cues of the dot stimuli in order to base their decision on numerical magnitude. This explanation was supported by Gilmore et al. (2013) who found that inhibitory control significantly predicted performance during the incongruent trials of the nonsymbolic numerical discrimination task, and explained the relationship between non-symbolic processing and arithmetic achievement in typically developing children. Although we did not explicitly examine inhibitory control in the current study, visuo-spatial working memory and inhibitory control have been found to be severely impaired in children with DD (Szucs et al., 2013a). As a result, deficits in either visuo-spatial working memory or inhibitory control (or both) would hinder their ability to access intact numerical representations (Szucs et al., 2013a, 2013b). This notion is supported by the strong correlation between visuospatial working memory and performance during the incongruent trials in children with DD. Children who had greater visuo-spatial working memory difficulties were unable to disambiguate between the non-numerical conflicting cues and numerical magnitude. To compensate for potential visuo-spatial working memory deficits (or potential inhibitory control deficits), children with DD require more time to resolve the conflict between numerical and non-numerical stimulus features to make successful non-symbolic numerical judgments.

Further support for this interpretation comes from methodological studies demonstrating that $w$ is highly influenced by task construction. Specifically, in a study conducted by Inglis and Gilmore (2013), it was demonstrated that $w$ changes depending on the stimulus duration despite differences in the onset to decision latencies in typically developing adults. More specifically, they found that $w$ decreases when the exposure to the stimulus display increases, therefore allowing participants time to access internal representations of quantity to accurately inform their decisions during more difficult trials. These findings clearly suggest that measures of ANS acuity are dependent on the time given to compare the displays of non-symbolic numerical magnitudes, thereby showing that measures like $w$ are not fixed internal variables, but are strongly modulated by stimulus-dependent processing. Given these findings, we speculate that if children with DD had more time to access numerical representations during trials where visual perceptual cues are incongruent with numerical 
magnitude, differences between children with DD and typically developing children would diminish. Furthermore, in view of our findings, we would predict that difficulties in processing rapidly presented non-symbolic stimuli would be more pronounced for children with DD who also have poor visuo-spatial working memory abilities. Given their limited capacity to hold nonsymbolic representations in working memory, shorter presentation times during incongruent trials would further augment their difficulties in choosing the numerically larger dot array.

Secondly, it is plausible that children with DD do have an impaired ANS, which forces them to compensate for numerical deficits by relying on the visual perceptual cues to perform the task. Put differently, when children with DD are unable to efficiently process numerical magnitude during dot discrimination, and they cannot rely on the visual properties of the stimuli to inform their decision during the incongruent trials, performance breaks down. However, during the congruent trials, area cues are consistent with the larger dot array and aid in the discrimination process. Therefore, it is plausible that children with DD who have ANS deficits are greatly influenced by visual perceptual processes when discriminating dot arrays. Although this explanation cannot be ruled out completely, visuo-spatial working memory modulates performance during non-symbolic numerical discrimination in children with DD. Therefore, a claim could be made that a non-symbolic numerical discrimination task is not a pure measure of numerical magnitude processing abilities. Furthermore, it highlights the role of visuo-spatial working memory during the reconciliation of visual perceptual cues and numerical magnitude whilst discriminating the incongruent dot arrays. Given how strongly visuo-spatial working memory predicts ANS acuity in children with DD, our data do not support the 'ANS hypothesis', particularly when ANS acuity is measured by a task previously assumed to measure pure numerical abilities.

Third, and lastly, it is conceivable that children with DD may experience both a weak ANS and suffer from the inability to inhibit non-numerical visual cues. More specifically, both imprecise approximate numerical representations and deficiencies in visuo-spatial working memory can explain the performance differences found in the incongruent trials between children with DD and typically developing controls. Rather than focusing on one core deficit causal theory of developmental dyscalculia, Fias, Menon and Szucs (2013) proposed that developmental dyscalculia is likely a multi-deficit disorder due to its heterogeneous nature and its high comorbidity rates with other learning disorders, such as dyslexia and ADHD (Lewis, Hitch \& Walker, 1994;
Shalev, Auerbach, Manor \& Gross-Tsur, 2000). The ability to perform arithmetic operations hinges on the competency of a complex system of cognitive processes such that it requires the abilities to process and access symbolic numerical magnitudes, and to temporarily store information during the manipulation of symbolic magnitudes in working memory. Research has shown that the recruitment of similar brain regions has been implicated during basic numerical processing tasks as well as visuospatial working memory tasks in typically developing populations (Dumontheil \& Klingberg, 2011; Zago \& Tzourio-Mazoyer, 2002). Indeed, these same regions show atypical activation patterns in children with DD (Rotzer, Loenneker, Kucian, Martin, Klaver et al., 2009; Price et al., 2007), which can compromise the efficiency of both cognitive systems. However, the dynamic relationships between numerical magnitude representations and working memory throughout development in children with DD remain unclear.

These findings have important implications for new avenues of research for investigating the core deficits of DD. For example, further research is required to understand how children with DD process conflicting non-numerical variables during dot discrimination. A closer examination into the role of visuo-spatial working memory and inhibitory control within the same sample of children with DD during the discrimination of different trial types is necessary to understand different compensatory mechanisms or strategies used by children with DD compared to typically developing children. Subsequently, these findings can lead to potential implications for training children with DD to focus on numerical magnitude while ignoring the irrelevant nonnumerical cues. We hypothesize that drawing their attention to the relationship between visual perceptual characteristics of objects (e.g. dot arrays) and numerical magnitude may build a solid understanding of numerical quantity. Thus, it would translate into better performance on tasks that require a thorough understanding of numerical magnitude, such as counting, estimation, and basic arithmetic.

To summarize, the present data demonstrate that visual stimulus properties influence performance on the non-symbolic numerical discrimination task in children with persistent $\mathrm{DD}$, specifically during trials where the visual perceptual cues conflicted with the numerically larger dot array. In addition, we found that individual differences in visuo-spatial working memory in children with DD modulated performance during the non-symbolic numerical discrimination, suggesting that children with DD rely on visuo-spatial processes to facilitate discrimination -more so during the incongruent trial types. Furthermore, the current study provides support 
for the notion that non-symbolic numerical discrimination tasks are unreliable measures of the integrity of numerical magnitude representations and raise the question of what underlying cognitive processes and strategies are employed during different trial types. It is important to note that discriminating dot arrays is a complex process that does not rely solely on approximate numerical representations, and future research is necessary to advance our understanding of the causal relationship between the ANS, visual perceptual cues, and visuo-spatial working memory in children with DD.

\section{Acknowledgements}

This research was supported by operating grants from the Natural Sciences and Engineering Research Council of Canada (NSERC) and the Canadian Institutes of Health Research (CIHR) to DA.

\section{References}

Alloway, T.P. (2007). Automated working memory assessment. London: Harcourt Publishing House Assessment.

American Psychiatric Association (2013). Diagnostic statistical manual of mental disorders (5th edn.). Arlington, VA: American Psychiatric Publishing.

Archibald, L.M.D., Oram Cardy, J., Joanisse, M.F., \& Ansari, D. (2013). Language, reading, and math learning profiles in an epidemiological sample of school age children. PloS ONE, 8 (10), e77463. doi:10.1371/journal.pone.0077463

Ashkenazi, S., Rosenberg-Lee, M., Metcalfe, A.W.S., Swigart, A.G., \& Menon, V. (2013). Visuo-spatial working memory is an important source of domain-general vulnerability in the development of arithmetic cognition. Neuropsychologia, 51 (11), 2305-2317. doi:10.1016/j.neuropsychologia.2013.06.031

Defever, E., Reynvoet, B., \& Gebuis, T. (2011). Task-and agedependent effects of visual stimulus properties on children's explicit numerosity judgments. Journal of Experimental Child Psychology, 116, 216-233.

Dehaene, S. (1997). The number sense. Oxford: Oxford University Press.

Dehaene, S. (2003). The neural basis of the Weber-Fechner law: a logarithmic mental number line. Trends in Cognitive Sciences, 7 (4), 145-147.

Dehaene, S. (2007). Symbols and quantities in parietal cortex: elements of a mathematical theory of number representation and manipulation. In P. Haggard, Y. Rossetti \& M. Kawato (Eds.), Sensorimotor foundations of higher cognition (pp. 527574). Cambridge, MA: Harvard University Press.

Dehaene, S., \& Cohen, L. (1995). Towards an anatomical and functional model of number processing. Mathematical Cognition, 1, 83-120.
De Smedt, B., \& Gilmore, C.K. (2011). Defective number module or impaired access? Numerical magnitude processing in first graders with mathematical difficulties. Journal of Experimental Child Psychology, 108 (2), 278-292. doi:10.1016/j.jecp.2010.09.003

Dumontheil, I., \& Klingberg, T. (2011). Brain activity during a visuospatial working memory task predicts arithmetical performance 2 years later. Cerebral Cortex, 22 (5), 10781085. doi:10.1093/cercor/bhr175

Duncan, G.J., Dowsett, C.J., Claessens, A., Magnuson, K., Huston, A.C., et al. (2007). School readiness and later achievement. Developmental Psychology, 43 (6), 1428-1446. doi:10.1037/0012-1649.43.6.1428

Fias, W., Menon, V., \& Szucs, D. (2013). Multiple components of developmental dyscalculia. Trends in Neuroscience and Education, 2 (2), 43-47. doi:10.1016/j.tine.2013.06.006

Fuhs, M.W., \& McNeil, N.M. (2013). ANS acuity and mathematics ability in preschoolers from low-income homes: contributions of inhibitory control. Developmental Science, 16 (1), 136-148. doi:10.1111/desc.12013

Gallistel, C.R., \& Gelman, R. (2000). Non-verbal numerical cognition: from reals to integers. Trends in Cognitive Sciences, 4 (2), 59-65.

Geary, D.C. (1993). Mathematical disabilities: cognitive, neuropsychological, and genetic components. Psychological Bulletin, 114 (2), 345-362.

Geary, D.C., \& Brown, S.C. (1991). Cognitive addition: strategy choice and speed-of-processing differences in gifted, normal, and mathematically disabled children. Developmental Psychology, 27 (3), 398-406. doi:10.1037//0012-1649.27. 3.398

Geary, D.C., Hoard, M.K., Byrd-Craven, J., \& DeSoto, M.C. (2004). Strategy choices in simple and complex addition: contributions of working memory and counting knowledge for children with mathematical disability. Journal of Experimental Child Psychology, 88 (2), 121-151. doi:10.1016/ j.jecp.2004.03.002

Gebuis, T., \& Reynvoet, B. (2012). The interplay between nonsymbolic number and its continuous visual properties. Journal of Experimental Psychology: General, 141 (4), 642648. doi:10.1037/a0026218

Gilmore, C., Attridge, N., Clayton, S., Cragg, L., Johnson, S., et al. (2013). Individual differences in inhibitory control, not non-verbal number acuity, correlate with mathematics achievement. PloS ONE, 8 (6), e67374. doi:10.1371/journal.pone. 0067374

Halberda, J., Mazzocco, M.M.M., \& Feigenson, L. (2008). Individual differences in non-verbal number acuity correlate with maths achievement. Nature, 455 (7213), 665-668. doi:10.1038/nature07246

Hanich, L.B., Jordan, N.C., Kaplan, D., \& Dick, J. (2001). Performance across different areas of mathematical cognition in children with learning difficulties. Journal of Educational Psychology, 93 (3), 615-626. doi:10.1037//00220663.93.3.615

Inglis, M., \& Gilmore, C. (2013). Sampling from the mental number line: how are approximate number system representations 
formed? Cognition, 129 (1), 63-69. doi:10.1016/j.cognition.2013.06.003

Jordan, N.C., Hanich, L.B., \& Kaplan, D. (2003). Arithmetic fact mastery in young children: a longitudinal investigation. Journal of Experimental Child Psychology, 85 (2), 103-119. doi:10.1016/S0022-0965(03)00032-8

Landerl, K., Bevan, A., \& Butterworth, B. (2004). Developmental dyscalculia and basic numerical capacities: a study of 8-9-year-old students. Cognition, 93 (2), 99-125. doi:10.1016/ j.cognition.2003.11.004

Leibovich, T., \& Henik, A. (2014). Comparing performance in discrete and continuous comparison tasks. Quarterly Journal of Experimental Psychology (2006), 67 (5), 899-917. doi:10.1080/17470218.2013.837940

Lewis, C., Hitch, G.J., \& Walker, P. (1994). The prevalence of specific arithmetic difficulties and specific reading difficulties in 9- to 10-year-old boys and girls. Journal of Child Psychology and Psychiatry, 35 (2), 283-292.

Libertus, M.E., Feigenson, L., \& Halberda, J. (2013). Numerical approximation abilities correlate with and predict informal but not formal mathematics abilities. Journal of Experimental Child Psychology, 116 (4), 829-838. doi:10.1016/j.jecp.2013.08.003

McLean, J.F., \& Hitch, G.J. (1999). Working memory impairments in children with specific arithmetic learning difficulties. Journal of Experimental Child Psychology, 74 (3), 240-260. doi:10.1006/jecp.1999.2516

Mazzocco, M.M.M., Devlin, K.T., \& McKenney, S.J. (2008). Is it a fact? Timed arithmetic performance of children with mathematical learning disabilities (MLD) varies as a function of how MLD is defined. Developmental Neuropsychology, 33 (3), 318-344. doi:10.1080/87565640801982403

Mazzocco, M.M.M., Feigenson, L., \& Halberda, J. (2011). Impaired acuity of the approximate number system underlies mathematical learning disability (dyscalculia). Child Development, 82 (4), 1224-1237. doi:10.1111/j.14678624.2011.01608.x

Mazzocco, M.M.M., \& Myers, G.F. (2003). Complexities in identifying and defining mathematics learning disability in the primary school-age years. Annals of Dyslexia, 53 (1), 218 253. doi:10.1007/s11881-003-0011-7

Mazzocco, M.M.M., \& Räsänen, P. (2013). Contributions of longitudinal studies to evolving definitions and knowledge of developmental dyscalculia. Trends in Neuroscience and Education, 2 (2), 65-73. doi:10.1016/j.tine.2013.05.001

Moyer, R.S., \& Landauer, T.K. (1967). Time required for judgements of numerical inequality. Nature, 215 (109), 15191520.

Mussolin, C., Mejias, S., \& Noël, M. (2010). Symbolic and nonsymbolic number comparison in children with and without dyscalculia. Cognition, 115 (1), 10-25. doi:10.1016/ j.cognition.2009.10.006

Nath, S., \& Szücs, D. (2014). Construction play and cognitive skills associated with the development of mathematical abilities in 7-year-old children. Learning and Instruction, 32, 73-80. doi:10.1016/j.learninstruc.2014.01.006
Passolunghi, M.C., \& Mammarella, I.C. (2012). Selective spatial working memory impairment in a group of children with mathematics learning disabilities and poor problem-solving skills. Journal of Learning Disabilities, 45 (4), 341-350.

Piazza, M. (2010). Neurocognitive start-up tools for symbolic number representations. Trends in Cognitive Sciences, 14 (12), 542-551. doi:10.1016/j.tics.2010.09.008

Piazza, M., Facoetti, A., Trussardi, A.N., Berteletti, I., Conte, S., et al. (2010). Developmental trajectory of number acuity reveals a severe impairment in developmental dyscalculia. Cognition, 116 (1), 33-41. doi:10.1016/j.cognition.2010. 03.012

Price, G.R., \& Ansari, D. (2013). Developmental dyscalculia. In O. Dulac, M. Lassonde \& H.B. Sarnat (Eds.), Handbook of clinical neurology (Vol 111, pp. 241-244). New York: Elsevier. doi 10.1016/B978-0-444-52891-9.00025-7

Price, G.R., Holloway, I., Räsänen, P., Vesterinen, M., \& Ansari, D. (2007). Impaired parietal magnitude processing in developmental dyscalculia. Current Biology, 17 (24), R1042R1043. doi:10.1016/j.cub.2007.10.013

Romano, E., Babchishin, L., Pagani, L.S., \& Kohen, D. (2010). School readiness and later achievement: replication and extension using a nationwide Canadian survey. Developmental Psychology, 46 (5), 995-1007. doi:10.1037/a0018880

Rotzer, S., Loenneker, T., Kucian, K., Martin, E., Klaver, P., \& von Aster, M. (2009). Dysfunctional neural network of spatial working memory contributes to developmental dyscalculia. Neuropsychologia, 47, 2859-2865.

Rousselle, L., \& Noël, M.-P. (2007). Basic numerical skills in children with mathematics learning disabilities: a comparison of symbolic vs. non-symbolic number magnitude processing. Cognition, 102 (3), 361-395. doi:10.1016/j.cognition.2006.01.005

Shalev, R.S., Auerbach, J., Manor, O., \& Gross-Tsur, V. (2000). Developmental dyscalculia: prevalence and prognosis. European Child \& Adolescent Psychiatry, 9 (Suppl. 2), 58-64.

Sowinski, C., LeFevre, J.-A., Skwarchuk, S.-L., Kamawar, D., Bisanz, J., et al. (2015). Refining the quantitative pathway of the Pathways to Mathematics model. Journal of Experimental Child Psychology, 131C, 73-93. doi:10.1016/j.jecp.2014.11.004

Swanson, H.L.E.E. (2004). Working memory and phonological processing as predictors of children's mathematical problem solving at different ages. Memory \& Cognition, 32 (4), 648661 .

Szucs, D., Devine, A., Soltesz, F., Nobes, A., \& Gabriel, F. (2013). Developmental dyscalculia is related to visuo-spatial memory and inhibition impairment. Cortex, 49 (10), 2674 2688. doi:10.1016/j.cortex.2013.06.007

Szucs, D., Devine, A., Soltesz, F., Nobes, A., \& Gabriel, F. (2014). Cognitive components of a mathematical processing network in 9-year-old children. Developmental Science, 17 (4), 506-524. doi:10.1111/desc.12144

Szücs, D., Nobes, A., Devine, A., Gabriel, F.C., \& Gebuis, T. (2013). Visual stimulus parameters seriously compromise the measurement of approximate number system acuity and 
comparative effects between adults and children. Frontiers in Psychology, 4, 444. doi:10.3389/fpsyg.2013.00444

Sekuler, R., Mierkiewicz, D., Sekuler, R., \& Mierkiewicz, D. (1977). Children's judgments of numerical inequality. Child Development, 48 (2), 630-633.

Wechsler, D. (1999). Wechsler abbreviated scale of intelligence. San Antonio, TX: Psychological Corporation.

Willcutt, E.G., Petrill, S.A., Wu, S., Boada, R., DeFries, J.C., et al. (2013). Comorbidity between reading disability and math disability: concurrent psychopathology, functional impairment, and neuropsychological functioning. Journal of Learning Disabilities, 46 (6), 500-516. doi:10.1177/ 0022219413477476

Wilson, A.J., \& Dehaene, S. (2007). Number sense and developmental dyscalculia. In D. Coch, G. Dawson \& K.W. Fischer
(Eds.), Human behavior, learning, and the developing brain: Atypical development (pp. 212-238). New York: Guilford Press. Woodcock, R.W., McGrew, K.S., \& Mather, N. (2001). Woodcock-Johnson Tests of Achievement. Itasca, IL: Riverside Publishing.

Zago, L., \& Tzourio-Mazoyer, N. (2002). Distinguishing visuospatial working memory and complex mental calculation areas within the parietal lobes. Neuroscience Letters, 331, 45-49.

Received: 21 November 2014

Accepted: 14 April 2015 